\title{
The Nature of Violence: Origins and Prevention of Healthcare Violence
}

\section{Sylvia Mcknight}

Nurse Educator, University of South Alabama, USA

*Corresponding author: Dr. Sylvia McKnight DNP, Nurse Educator, University of South Alabama, USA, Tel: [254] 214-7697; E-mail: smcknight541@gmail.com

Received date: June 24, 2014, Accepted date: July 14, 2014, Published date: July 17, 2014

Copyright: @ 2014 Mcknight S. This is an open-access article distributed under the terms of the Creative Commons Attribution License, which permits unrestricted use, distribution, and reproduction in any medium, provided the original author and source are credited.

\begin{abstract}
Violence in healthcare is a prominent global concern. To prevent violence it is necessary to have an organized plan of action against violence. It is vital to learn where violence originates and then to develop an organized interventional plan to prevent violence. This article analyzes the origins [causes and risk factors] of violence and delineates the process and development of an evidence based violence prevention plan designed to negate violence risk factors and prevent violence. Specific techniques are presented for prevention and de-escalation of aggression to prevent violence. Strategies are determined for development and implementation of effective violence prevention plan utilizing holistic evidence based interventions to prevent/reduce healthcare violence for a safe and therapeutic environment for everyone.
\end{abstract}

Implications for practice are strategies for best practice in safety and violence prevention. Environmental, patient and caregiver interventions for prevention of aggression and violence in healthcare facilities is delineated as well as psychosocial management of aggressive behavior for safety in the healthcare environment. An effective violence prevention educational plan is presented for holistic applications for decreasing healthcare violence promoting mental health, wellness and recovery.

Keywords: Violence risk factors; Anger; Aggression; Agitation; Violence; Psychiatric illness; Organic mental illness; Assessment; Early intervention; De-escalation; Violence prevention initiatives; Safety; Mental health; Wellness and recovery

\section{Introduction}

Violence in mental health facilities is a prominent global healthcare concern. The World Health Organization (WHO) in 2002 recognized violence as a global health priority with significant health, social and economic consequences. Anger, aggression and violence are everywhere. Violence permeates every aspect of our culture, including mental health care organizations [1]. It is estimated that 50 percent of healthcare workers will encounter violence at least once in their careers [2]. Employees in mental health facilities face violence very often [3]. It is estimated that every year one in four mental health nurses suffers a disabling injury from patient violence [4]. An essential safety strategy for violence prevention is the development and implementation of an organized violence prevention plan for mental health care facilities. A violence prevention plan decreases violence and assists to maintain a safe and therapeutic environment for everyone. This article describes the process of the development of an evidence based violence prevention plan with interventional strategies effective to prevent/reduce violence in mental health facilities. The violence prevention plan developed is based on violence origins [causes] and prevention of violence, focusing interventional strategies to prevent the phenomenon of violence in mental health facilities promoting safety, health, wellness and optimal recovery outcomes.

\section{Background}

To prevent violence it is necessary to learn where violence originates and determine if violence is natural to the human species.
There are many theories on the origins of violence in human culture. History tells us that human life in centuries past was nasty, violent, brutish and short. It has been proposed that life in ancient times was not always harsh and violent. Demeo published substantial proof that our ancestors were peaceful, non-violent and far more social than they are today. Demeo's proposed that climate change around 4000 BC brought hardship, famine, starvation and migrations which forced humans into violent social patterns over competition for scarce resources for basic survival. These oppressive ecological conditions brought deprivation causing conflict, warfare, and violence to first appear among the ancient peoples. Violence is not natural to the human species [5]. Violence does not arise from the human character. Violence is an aberrant form of behavior for all the human species. History teaches us that many times violence originates from unmet basic human needs for survival. In primate studies it is also noted that both violence and peaceful behavior can be learned [6,7]

Violence is defined as an outburst of physical force that abuses, injures or harms another individual or object [8]. The concepts of violence can exist in a continuum. Littrell \& Littrell proposed that a linear progression or continuum may better conceptualize the various components of violence. Appropriate organized healthcare response to the phenomenon of violence requires recognition that violence originates from multiple causes. There is robust evidence that the cause of violence is associated with patient risk factors, environmental risk factors, caregiver [nursing and mental health staff] risk factors and that in most cases violence is predictable [9].

\section{Method}

The process of developing an evidence based violence prevention plan begins with researching each of the violence cause risk groups of patient, environment and caregiver for risk factors associated with the violence as well as researching effective strategies to negate each 
violence risk factor and prevent violence [10]. Extensive research was completed utilizing multiple data bases such as PubMed and CINAHL from years 1989 to 2014. The research identified definitive risks for violence [2]. The research base contained iAfter each group's risk factors is identified then educational strategies and an interventional plan is developed from best practice to reduce/eliminate each risk for violence.

\section{Patient violence risk factors}

Literature review on patient risk factors for violence indicates there are primary groups of patient factors that are associated with increased risk of violence. The first high risk group for violence are those individuals with a diagnosis of psychosis such as schizophrenia or bipolar disorder [11]. The second high risk group for violence is organic patients with neurological or medical disorders such as brain injury, dementia, organic brain syndrome, alcohol or drug intoxication, infections, delirium or degenerative diseases of brain. The last high risk group for violence is personality disorders such as borderline personality disorders known for poor impulse control [12].

Mental instability in the individual increases the risk of directed violence toward others. Indicators for primary motivation for violence include 1) disordered impulse control, 2) psycopathy and 3) symptoms of psychosis [4]. The presence of delusions or command hallucinations with violent content is an indicator of greatly increased risk of violence in that individual. Belonging to a demographic group with an increased prevalence of violence such as young and male also increases the violence risk $[13,14]$.

Patient risk factors that present consistent risk for impending violence include committing a recent act of violence. Violent people tend to be repeaters of violence with over 77 percent of inpatient violence perpetrated by individuals with a history of violent assault. The majority of inpatient violence is associated with a primary diagnosis of schizophrenia 62.6\% and Bipolar Disorder 24.6\% [15,16].

Patient risk factors for violence include an agitated state with anger or disorganized thought processes. If the patient has a detailed or planned threat for violence the risk for a violent act by that individual is increased [11,17]. If the individual has an available means for inflicting injury such as ownership of a weapon he/she is more at risk to use that weapon to commit a violent act. Carrying objects that may be used as weapons such as a chair are an indicator of increased risk of violence with that object.

Indicators of increased risk of violence include progressive psychomotor agitation, irritability and paranoid symptoms. Substance abuse and/or withdrawal symptoms can trigger aggression in inpatients. Overcrowding, frustration and boredom can cause aggression toward others [18]. Medication toxicity or allergic reactions to medications can cause excited delirium and agitated/aggressive states that may result in violent behavior toward others [19].

\section{Environmental violence risk factors}

Environmental risk factors may contribute substantially to the risk of violence. Research suggests that environmental factors such as levels of stimulation can affect the risk for violence. Environmentent factors such as excessive external stimuli such as noise or physical activity is associated with violent behavior. An environment that is devoid of therapeutic groups and activities contributes to boredom and increased risk of episodes of violence. Environment factors such as the unpredictable behavior of other patients in the environment may increase the risk of inpatient violence. Changing environments such as during remodeling, renovation or construction during may increase risk of violence of inpatients [13].

Environmental risks may include limited space due to shared rooms and lack of privacy [20]. A major contributing environmental factor is lack of unit structure and a predictable schedule. The most common times for inpatient violence are at meal times when patients have eating utensils and during shift change when the least amount of staff are available on the units. The environmental factors of an influx of new admissions especially male and involuntary patients poses an increased risk factor of violence. Lack of autonomy such as a locked door and involuntary admission, or being prevented from leaving the hospital is a major indicator of increased risk of inpatient violence $[21,22]$.

\section{Caregiver violence risk factors}

Evidence based research on caregiver violence risk factors indicates caregiver characteristics greatly contribute to the risk factors for inpatient violence. The educational level of the caregiver staff member impacts risk of encountering violence. Staff members that attend education on violence prevention reduce their risk of becoming a victim of violence [15]. It is noted that the higher the educational level of the nurse, the lower the risk for encountering violence. Nurse aides and nursing students have the highest risk for encountering physical violence and physical assault.

The caregivers work experience effect the risk of a violent encounter. Skills and experience play a critical role in violence prevention. Newly hired nursing and mental health staff encounter more violence than seasoned staff. Unfamiliarity of staff with the patient and the venue of care increase the risk of a violent encounter. A lack of flexibility and rigidity of adhering to routines may trigger violence and assaults [4]. Assaults frequently occur while staff members are administering medications or placing a patient in a restraint.

Caregiver attitude and skill impact violence risk. Increased episodes of inpatient violence correlate with caregiver ineffective therapeutic communication skills, detached, aloof or a superior manner. The caregiver inability to listen and lack of training in aggression management increase violence risk [23]. Caregivers that are less available to patients both physically and emotionally such as those not being available to listen are more apt to encounter violence.

Caregiver and patient interaction styles that factors affect violence risk are many. They include caregivers that initiate power struggles and/or are very emotional such as speaking in a loud tone of voice, anxious or over-reacting to situations [14]. Caregivers that place too many demands on a patient at one time may cause the patient to feel powerless and increases the risk of aggression and violence in that individual.

\section{Analysis}

The violence prevention plan is developed in a three domain framework of patient, environment and caregiver. The violence risk factors were delineated and separated by each domain. After the risk factors were divided the violence prevention interventional strategies were also separated by patient, environment and caregiver. Each risk factor was then paired with the most effective interventions to negate 
Page 3 of 6

each violence risk factor. The educational interventions were then structured into an interventional framework that formed the violence prevention educational plan. The completed violence prevention plan is listed in Appendix 5. The educational program was then implemented as an organized plan to reduce inpatient violence.

\section{Patient factors admission risk assessment for safety}

To address patient risk factors for violence it is necessary to understand and acknowledge that violence is a common reason for psychiatric evaluation and admission to a mental health facility. Over $10 \%-40 \%$ of mental health admissions are admitted to address aggression and violence issues [24,25]. Many patients arrive to medical and mental health facilities with existing aggression and violence issues as reasons for admission to mental health facilities. Clinicians should understand the causes, as well as risk factors of violence and initiate early intervention on admission to prevent violence. Research indicates violence prevention begins the first day a patient is admitted with an effective risk assessment [26-28].

Strong clinical admission assessment is one of the most vital interventions to prevent healthcare violence [25]. It is imperative that an efficient violence prevention plan include a risk assessment on admission as a critical part of a comprehensive mental health assessment. Violence prevention includes professionals routinely assess, document and monitor dynamic risk factors for violence [29]. An admission risk assessment based on Joint Commission recommendations is listed in Appendix 1. Risk assessments increase staff awareness and safety to anticipate and potentially prevent aggression and violence [14,21]. It is important to include risk factors for assault and violence in the initial evaluation of the patient and ongoing [30]. Vital assessment as seen in Appendix 2 identifies the situations which trigger a patient's violent response. The admission assessment on violence triggers (Appendix 2) assists in identifying precursors to violence that may be diminished or eliminated from the environment to prevent episodes of violence or self harm until the patient becomes emotional stable [31].

An effective admission assessment includes a de-escalation assessment completed on admission as a critical part of a comprehensive mental health assessment [29,32]. An example of a deescalation assessment is seen in Appendix 3. The purpose of the deescalation interventions (Appendix 3) assessment is to obtain vital information from the patient on personal preferences for interventions that are most effective in calming when agitated or depressed to prevent harming behaviors. It is important to integrate all the assessment factors into the treatment plan.

\section{Caregiver factors de-escalation training for violence prevention}

Evidence based research indicates that early intervention and deescalation training of mental health professionals greatly reduces the risk of healthcare violence $[31,33]$. De-escalation is defined as the art of calming an individual utilizing therapeutic communication techniques and interventions that will diffuse an agitated state [14]. Appendix 4 lists therapeutic de-escalation techniques to reduce/ eliminate agitated states and prevent violence greatly reduces the risk of violence.

\section{Early intervention for violence prevention}

Research indicates that early identification of escalating behaviors and immediate intervention Including early intervention and deescalation training as part of a violence prevention educational program will greatly impact in violence reduction. De-escalation techniques listed in Appendix 4 include encouraging verbalization such as asking "what is upsetting you?" or "tell me how it started?", "What were the precipitating events?" These open ended questions encourage verbalization of anger instead of acting out the anger by physical aggression toward others. Talking with staff, family or friends will assist in calming the individual and reduce the risk of violence $[31,33,34]$. These therapeutic interventions can effectively diffuse an agitated state.

Violence may begin from a calm state then escalate to anxious, agitated, aggressive and then violent behavior. Research indicates it is imperative that early assessment and de-escalation interventions be implemented quickly to break the aggression cycle and prevent violence [11]. Training of all mental health staff in de-escalation techniques is critical to prevent inpatient violence. Implementation of a mental health learning needs assessment is beneficial in ascertaining healthcare staff safety knowledge deficits and to develop de-escalation educational training to meet these educational needs.

To prevent violence it is necessary to intervene and defuse the situation before the patient gets to the point of loss of all self-control. Adequate acuity based unit staffing is important as staff need to be available to intervene early with de-escalation interventions to prevent violence. It is vital that healthcare staff intervene at the first sign that a client is escalating [35]. Decrease environmental stimulation if possible and move the agitated individual to a calmer place [sensory or comfort room if available]. Acknowledge, validate and encourage the individual to discuss his/her feelings. Educate the individual on stress management techniques [36]. Focus on elements of treatment plan to prevent violence, eg. de-escalation, prn medication, special observation one-to-one, and coping skills development [37]. If these least restrictive alternatives fail the last resort for violence prevention is seclusion or application of a physical restraint per hospital policy.

\section{Caregiver factors minimizing personal risk}

The literature review indicates that staff education and training in interpersonal skills is effective for improving safety and violence prevention. Education and training programs in communication skills of empathy, listening, and low expressed emotion to role model calmness greatly reduces the risk of patient violence [38]. One of the best interventions for preventing violence is for mental health staff to attend and participate in education focused on violence prevention [techniques both physical and verbal] to create a positive therapeutic alliance to develop rapport as well as prevent violence. Education on maintaining reliability and consistency in therapeutic care improves safety and outcomes of care [14,15,21]. Provide staff with clinical supervision to review interactions with patients such as debriefing after acting out events and to review techniques as well as strategies to prevent future violence.

As a healthcare professional interacting with patients daily it is important to implement strategies to decrease personal risk for encountering violent behavior. For safety utilize nonthreatening body language such as opening the hands to expose the palms. Respect the patient's personal space and boundaries by keeping an adequate personal space when interacting [12,39]. Avoid standing closely or 
over (within reach) of an angry agitated or threatening patient. Healthcare staff should position themselves so they have immediate access to the door of the room in case there is a need to leave room quickly to obtain assistance. Choosing to leave the door open while conversing with a patient is important for personal safety. For safety always know where your colleagues are in the environment. Remember to never become isolated with an agitated person.

It is important to avoid personal hazards such as long hair. Hair worn short or in a bun is safer than long hair. It is safer to avoid wearing clothing with potential for harm such as dangle earings, scarves, neck ties, heavy necklaces and to avoid wearing a stethoscope around the neck $[40,41]$.

\section{Environmental factors changing healthcare culture}

Research suggests that hospital environments affect patient behavior in either a positive or negative manner. To prevent inpatient violence it is important to address environmental considerations [42]. Promotion of a healing environment with therapeutic activities and surroundings benefit in reducing episodes of violence. It is important to reduce and mitigate risk during times of transition. It is vital for staff to be available for therapeutic intervention and de-escalation even during shift change [14]. The staggering of nursing staff shift schedules is an important environmental intervention to prevent gaps of care during shift changes. If is important that staff be available for therapeutic intervention and de-escalation even during shift changes. It is beneficial to schedule activities on weekends, evenings and during shift changes. Create comforting and calming physical environments [42]. Encourage staff to increase time staff spent on the unit observing, assessing and interacting with patients to encourage therapeutic rapport and prevent violence.

\section{Environmental factors improving milieu structure}

Environmental interventions for violence prevention include development of a unit culture that provides meaningful and predictable unit activities. Implementing psycho-education groups and coping with symptoms groups to aid in the recovery process. Unit activities and groups with structure and schedules posted and given to patients as well as consistency in rules and staff roles are beneficial [43]. Identify incentives for optimal improvement. Promote peer advocates as mentors and facilitators of patient centered care [44]. Have consistency in staff implementation of therapies and unit organization. Maximize therapeutic group activities to include all patients if possible. Plan a conflict resolution group to voice and air issues before open conflict erupts. Introduce an anger management group to assist in self control of aggressive impulsive behavior. An exercise group is beneficial to release endorphins which are the bodies natural calming hormones to restore homeostasis.

\section{Environmental factors safety in milieu control}

The contraband hazardous item search is important to maintain a safe therapeutic milieu for the patient and everyone [45]. All patients and their belongings are searched for hazardous items when entering an inpatient mental health unit [38]. A very careful head to toe body pat search and a metal detector scan is completed by mental health staff in most mental health facilities for prevention of dangerous items entering the unit for safety.

Violence and assaults are more common in high traffic or high patient density areas. It is critical to avoid large numbers of patients congregating without staff control such as in lines for medication, showers, or telephone access [31]. Control patient access to high risk areas where staff enter/leave or traffic through the unit. Debrief after violence episodes to learn techniques to prevent future violence.

Music and television content affect the level of acuity of the treatment milieu. It is important to have television programs that are therapeutic. Monitor, control and avoid violent television programs. Relaxation music is beneficial for reducing anxiety and promoting a calming milieu.

\section{Environmental factors psychosocial management of aggression}

One of the most vital interventions for management of aggression to prevent violence is direct communication between patient, provider and staff. Utilizing clear open communication can resolve misunderstandings before it becomes a behavioral issue [26]. Be an advocate for the patient and available for communication to talk out issues before they become a behavior problem.

It is important the patient be able to correctly interpret communication. Minimize any sensory deficits by providing eyeglasses and a hearing aid $[20,39]$. Always individualize the treatment plan to focus on each patients pertinent needs. Emphasize the patient strengths and the hope of recovery. Collaborate with patients in treatment planning. Be flexible with creative treatment combinations to promote holistic healing and violence prevention.

\section{Results}

The application of the violence prevention plan includes holistic interventions for the patient, environment and caregiver to substantially decrease inpatient violence. The suggested violence prevention plan when implemented in an inpatient mental health setting over a nine month period of time resulted in a substantial reduction of inpatient violence rates by $100 \%$. When fully implemented the violence prevention plan effectively reduced inpatient violence rates to zero for the first time in nearly a decade. The program and training had an additional advantage of also eliminating restraint use and resulted in a restraint free facility.

\section{Discussion}

The violence prevention plan suggested and listed in Appendix 5 addresses the critical areas of violence origins for effective strategies to prevent violence in mental health facilities for safe holistic care. A violence prevention plan addressing the holistic risk factor domains of patient, caregiver, and environment are very effective as an organized method to reduce/prevent healthcare violence. The violence prevention plan suggested is a comprehensive therapeutic program educating and implementing best practice in violence prevention for a safe healthcare environment.

The disadvantage of the violence prevention plan is that it implements comprehensive change. The complete implantation time of the plan was 9 months of tiered education and training in violence techniques as well as environmental changes. The plan introduced effective patient assessment as well as healthcare staff and patient education. Environmental changes are extensive to create a caring and healing environment for recovery oriented care. The nursing and mental health staff education suggested for violence prevention is indepth and requires educational time off the units for training. 
The major advantage of the violence prevention program is it empowers holistic applications for safety. Healthcare violence has more than one point of origin. Holistic applications utilizing the framework of patient, environment and caregiver comprehensively addresses the multiple origins of violence for effective interventions and strategies to prevent/reduce healthcare violence.

\section{Conclusion}

Violence in mental health facilities is preventable with early assessment and interventional strategies for safety. Violence prevention educational plans targeting the causes of inpatient violence will greatly improve safety for patients, staff and all individuals in the therapeutic mental health care environment. Safety is improved with holistic application of interventions focused on the patient, caregiver and environmental risk factors to prevent violence. Holistic applications for violence prevention will greatly benefit everyone and is truly the future of safe mental health care. Implementation of a violence prevention plan will improve care and provide a safe therapeutic mental health environment for everyone. Research is needed into advanced application of violence prevention plans into all healthcare environments. The violence prevention plan is versatile, improves safety, and can provide optimal recovery outcomes, improving the lives of vulnerable populations promoting health, wellness, and recovery.

\section{References}

1. Liss GM, McCaskell L [1994] Violence in the workplace. CMAJ 151: 1243-1246.

2. Findorff MJ, McGovern PM, Wall M, Gerberich SG, Alexander B [2004] Risk factors for work related violence in a health care organization. Inj Prev 10: 296-302.

3. Franz S, Zeh A, Schablon A, Kuhnert S, Nienhaus A [2010] Aggression and violence against health care workers in Germany--a cross sectional retrospective survey. BMC Health Serv Res 10: 51.

4. Simon R, Tardiff [2008] Violence Assessment and Management. Washington: American Psychiatric Publishing.

5. Pinker S [2011] The Better Angels of Our Nature Why Violence Has Declined. New York: Penguin Books.

6. Copeland-Linder N, Lambert SF, Ialongo NS [2010] Community violence, protective factors, and adolescent mental health: a profile analysis. J Clin Child Adolesc Psychol 39: 176-186.

7. Sapolsky R [2007] Peace among primates. Greater Good, 1-5.

8. Sunderland T [1997] The diagnosis and epidemiology of violence and agitation. American Psychiatric Association 278: 1363-1371.

9. Bowers L, Allan T, Simpson A, Jones J, Van Der Merwe M, et al. [2009] Identifying key factors associated with aggression on acute inpatient psychiatric wards. Issues Ment Health Nurs 30: 260-271.

10. Distasio CA [1994] Violence in health care: institutional strategies to cope with the phenomenon. Health Care Superv 12: 1-34.

11. Hodgins S [2008] Violent behaviour among people with schizophrenia: a framework for investigations of causes, and effective treatment, and prevention. Philos Trans R Soc Lond B Biol Sci 363: 2505-2518.

12. Umhau JC, Trandem K, Shah M, George DT [2012] The physician's unique role in preventing violence: a neglected opportunity? BMC Med 10: 146 .

13. Chou KR, Kaas MJ, Richie MF [1996] Assaultive behavior in geriatric patients. J Gerontol Nurs 22: 30-38.

14. Hamrin V, Iennaco J, Olsen D [2009] A review of ecological factors affecting inpatient psychiatric unit violence: implications for relational and unit cultural improvements. Issues Ment Health Nurs 30: 214-226.
15. Chou KR, Lu RB, Mao WC [2002] Factors relevant to patient assaultive behavior and assault in acute inpatient psychiatric units in Taiwan. Arch Psychiatr Nurs 16: 187-195.

16. Falk O, Wallinius M, Lundström S, Frisell T, Anckarsäter H, et al. [2014] The $1 \%$ of the population accountable for $63 \%$ of all violent crime convictions. Soc Psychiatry Psychiatr Epidemiol 49: 559-571.

17. Glasser W [1997] Violence and aggression: Assessing risk, affecting outcomes.

18. Cole A [2005] Four in five nurses on mental wards face violence. BMJ 330: 1227.

19. Owen C, Tarantello C, Jones M, Tennant C [1998] Repetitively violent patients in psychiatric units. Psychiatr Serv 49: 1458-1461.

20. Negley EN, Manley JT [1990] Environmental interventions in assaultive behavior. J Gerontol Nurs 16: 29-33.

21. Johnson M, Delaney K [2007] Keeping the unit safe: The anatomy, of escalation. Journal of the American Psychiatric Nurses Association, 13: $42-51$.

22. Lehmann LS, McCormick RA, Kizer KW [1999] A survey of assaultive behavior in Veterans Health Administration facilities. Psychiatr Serv 50: 384-389.

23. Lanza ML, Kayne HL, Hicks C, Milner J [1991] Nursing staff characteristics related to patient assault. Issues Ment Health Nurs 12: 253-265.

24. Swanson JW [2008] Preventing the unpredicted: managing violence risk in mental health care. Psychiatr Serv 59: 191-193.

25. Singh JP, Fazel S, Gueorguieva R, Buchanan A [2014] Rates of violence in patients classified as high risk by structured risk assessment instruments. Br J Psychiatry 204: 180-187.

26. American Psychiatric Association [2007] American Psychiatric Nurses Association and National Association of Psychiatric Health Systems

27. Gately L and Stabb S [2005] Psychology students training in the management of potentially violent clients. Professional Psychology Research \& Practice 36: 681-687.

28. Haggård-Grann U, Hallqvist J, Långström N, Möller J [2006] Short-term effects of psychiatric symptoms and interpersonal stressors on criminal violence--a case-crossover study. Soc Psychiatry Psychiatr Epidemiol 41: 532-540.

29. McKnight S [2011] Risk assessment in the electronic age: Application of the circle of caring model. Online Journal of Nursing Informatics 15.

30. Abderhalden C, Needham I, Dassen T, Halfens R, Haug HJ, et al. [2008] Structured risk assessment and violence in acute psychiatric wards: randomised controlled trial. Br J Psychiatry 193: 44-50.

31. Chabora N, Judge-Gorny M, Grogan K [2003] The Four S Model in action for de-escalation. An innovative state hospital-university collaborative endeavor. J Psychosoc Nurs Ment Health Serv 41: 22-28.

32. Singh JP, Grann M, Lichtenstein P, Långström N, Fazel S [2012] A novel approach to determining violence risk in schizophrenia: developing a stepped strategy in 13,806 discharged patients. PLoS One 7: e31727.

33. United States Department of Health and Human Services [2007] Roadmap to seclusion and restraint free mental health services. United States Department of Health and Human Services.

34. Hilgers J [2003] Comforting a confused patient. Nursing 33: 48-50.

35. Biancosino, Delmonte, Grassi, S, Miglio R, Girolamo G [2009] Violent behavior in acute psychiatric inpatient facilities: A national survey in italy. Journal of Nervous and Mental Disorders 197.

36. Seaward B [2006] Managing Stress, [5thedn], Boston: Jones and Bartlett Publishers.

37. Timko C, Valenstein H, Lin PY, Moos RH, Stuart GL, et al. [2012] Addressing substance abuse and violence in substance use disorder treatment and batterer intervention programs. Subst Abuse Treat Prev Policy 7: 37.

38. Rice M, Grant T, Barney G, Quinsey V [1989] Violence in Institutions, Prediction and Control. Toronto: Hogrete and Huber Publishers.

39. Sifford-Snellgrove KS, Beck C, Green A, McSweeney JC [2012] Victim or initiator? Certified nursing assistants' perceptions of resident 
Citation: Mcknight S (2014) The Nature of Violence: Origins and Prevention of Healthcare Violence. J Nurs Care 3: 183. doi: 10.4172/2167-1168.1000183

Page 6 of 6

characteristics that contribute to resident-to-resident violence in nursing homes. Res Gerontol Nurs 5: 55-63.

40. Boyd M [2008] Psychiatric Nursing. Philadelphia: Wolters Klumer/ Lippincott Williams \& Wilkins.

41. Benveniste KA, Hibbert PD, Runciman WB [2005] Violence in health care: the contribution of the Australian Patient Safety Foundation to incident monitoring and analysis. Med J Aust 183: 348-35

42. Felgen J [2004] A caring and healing environment. Nurs Adm Q 28: 288-301.

43. Adamson MA, Vincent AA, Cundiff J [2009] Common ground, not a battle ground. Violence prevention at a detoxification facility. J Psychosoc Nurs Ment Health Serv 47: 28-35.
44. Henry DB, Miller-Johnson S, Simon TR, Schoeny ME; Multi-site Violence Prevention Project [2006] Validity of teacher ratings in selecting influential aggressive adolescents for a targeted preventive intervention. Prev Sci 7: 31-41.

45. Damon L, Matthew J, Sheehan L, Uebelacker L [2012] Inpatient Psychiatric Nursing: Clinical Strategies \& Practical Interventions. New York: Springer Publishing. 